\title{
Editorial
}

\section{Recent Trends in Boundary Value Problems 2014}

\author{
Bashir Ahmad, ${ }^{1}$ Juan J. Nieto, ${ }^{1,2}$ Donal O'Regan, ${ }^{3}$ and Agacik Zafer ${ }^{4}$ \\ ${ }^{1}$ Department of Mathematics, Faculty of Science, King Abdulaziz University, P.O. Box 80203, Jeddah 21589, Saudi Arabia \\ ${ }^{2}$ Department of Mathematical Analysis, Faculty of Mathematics, University of Santiago de Compostela, \\ 15782 Santiago de Compostela, Spain \\ ${ }^{3}$ School of Mathematics, Statistics and Applied Mathematics, National University of Ireland, Galway, Ireland \\ ${ }^{4}$ College of Engineering and Technology, American University of the Middle East, Block 3, Egaila, Kuwait \\ Correspondence should be addressed to Bashir Ahmad; bashirahmad_qau@yahoo.com
}

Received 7 October 2014; Accepted 7 October 2014

Copyright (C) 2015 Bashir Ahmad et al. This is an open access article distributed under the Creative Commons Attribution License, which permits unrestricted use, distribution, and reproduction in any medium, provided the original work is properly cited.

This special issue is a continuation of the earlier one entitled Recent Trends in Boundary Value Problems published in 2013. The importance of boundary value problems emerges from its continuous theoretical development and its wide applicability to a variety of problems of practical nature. Such problems involve different kinds of equations such as ordinary, partial, functional, fractional, and integrodifferential equations and the boundary data varying from twopoint and periodic to multipoint and nonlocal boundary conditions. Well-posed and ill-posed, local and nonlocal, linear and nonlinear, singular and nonsingular, and free and fixed problems are important and interesting types of boundary value problems.

This special issue is mainly concerned with new and recent developments in the theory, methods, and applications of boundary value problems and, as requested in the call for papers, includes papers on existence and uniqueness of solutions, analytical methods, iterative methods, perturbation techniques, numerical techniques, applications in physical sciences, applications in engineering, biology, and medicine, applications in economics and social sciences.

More precisely, the current issue includes research articles addressing finite element algorithm for Laplace eigenvalue problems; discontinuous Galerkin immersed finite volume method for anisotropic flow models; application of variant fountain theorems to impulsive Dirichlet boundary value problems; computation of eigenpairs of two-parameter Sturm-Liouville problems via regularized sampling method; similarity solutions for nonlinear partial differential equations; semi-Fredholm solvability for ProtterMorawetz problem; inverse acoustic scattering problem; fractional order model of dengue fever; nonlinear nonlocal systems of reaction-diffusion equations; existence results for boundary value problems involving singularity on the boundary, second-order singular impulsive equations, third-order equations with four-point boundary conditions, impulsive difference equations with antiperiodic boundary conditions, higher dimensional systems of fractional finite difference equations, and boundary value problems of fractional differential equations. Contribution for this special issue comes from different parts of the world (Bulgaria, China, France, Greece, Iran, Pakistan, Qatar, Russia, Saudi Arabia, Spain, Thailand, Turkey, UK, and USA).

Evidently, it is not possible to cover all aspects of current research on boundary value problems adequately in a special issue, but we believe that it reflects both theoretical research and potential applications including recent challenging problems, new ideas, and open problems.

\section{Acknowledgments}

The Guest Editors of this special issue would like to express their gratitude to the authors for their contributions, which have made it possible to have a very stimulating interchange of ideas. They are also thankful to the individuals who served as referees for the submitted manuscripts. It is hoped that the topics covered in this special issue will enhance and motivate future research in several directions.

Bashir Ahmad Juan J. Nieto Donal O'Regan Agacik Zafer 


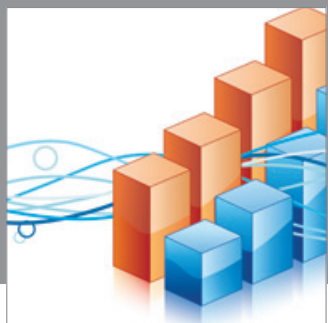

Advances in

Operations Research

mansans

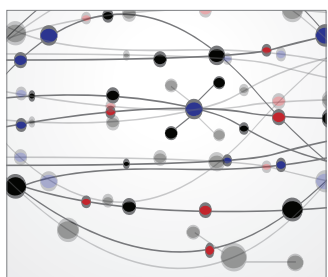

The Scientific World Journal
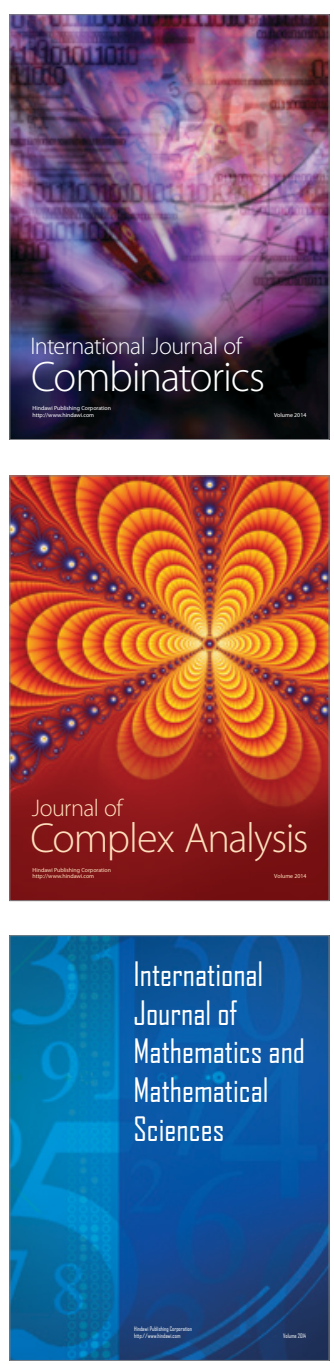
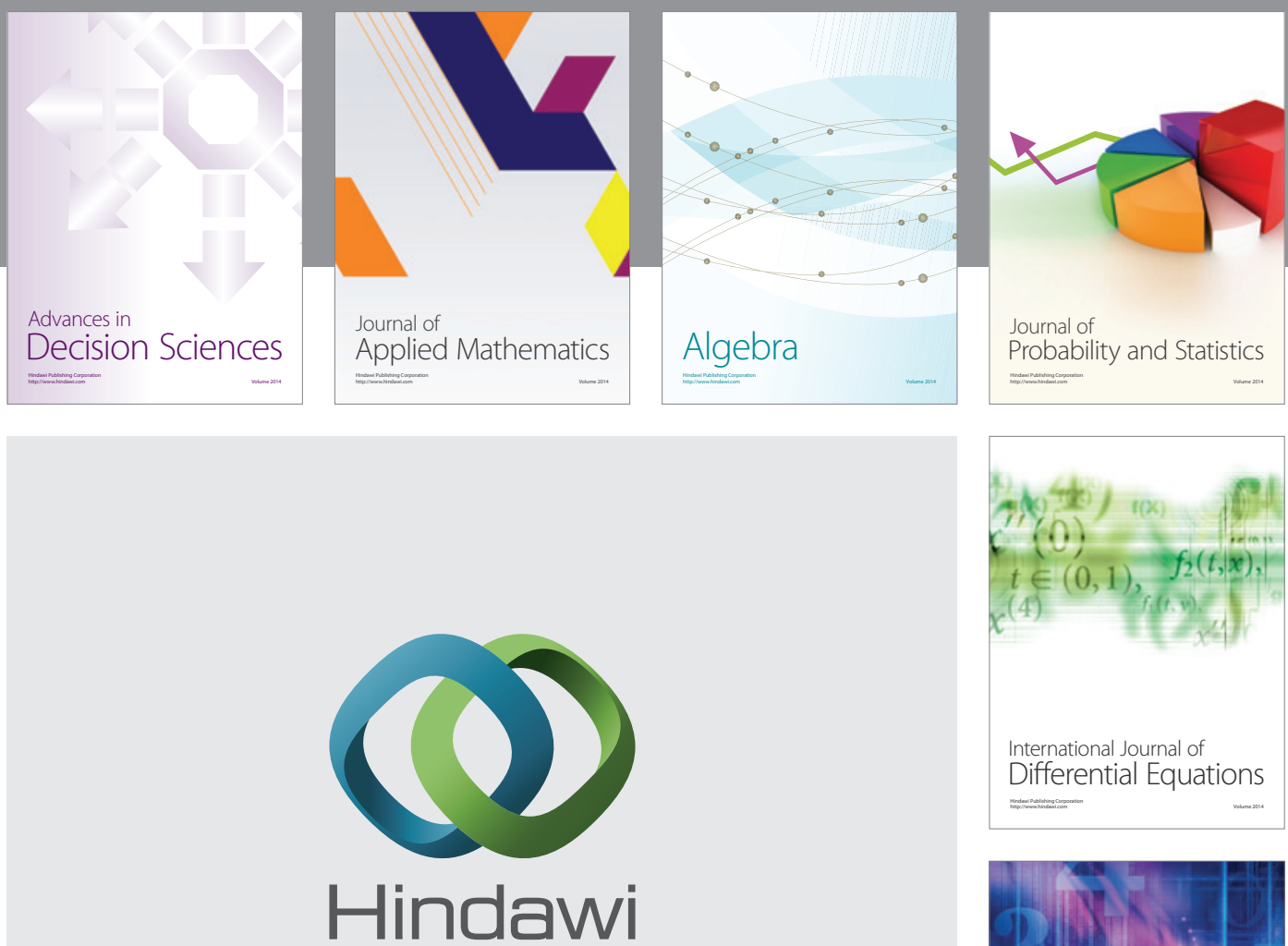

Submit your manuscripts at http://www.hindawi.com
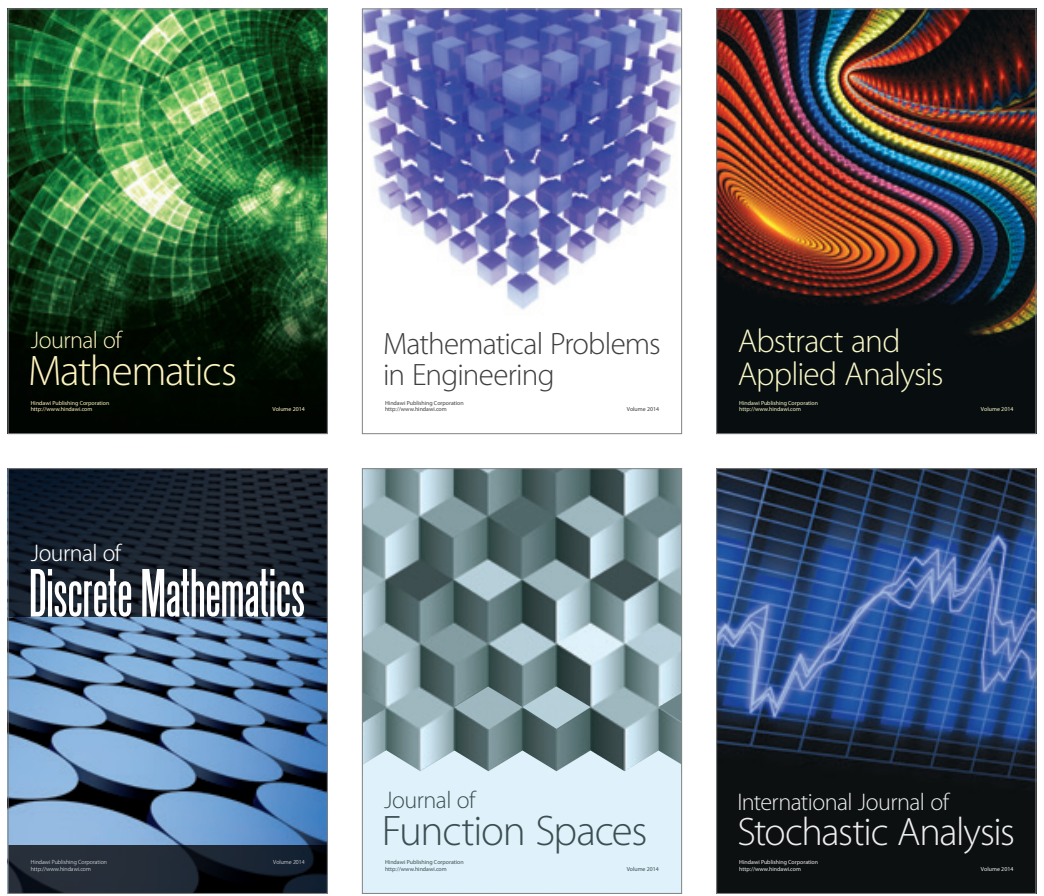

Journal of

Function Spaces

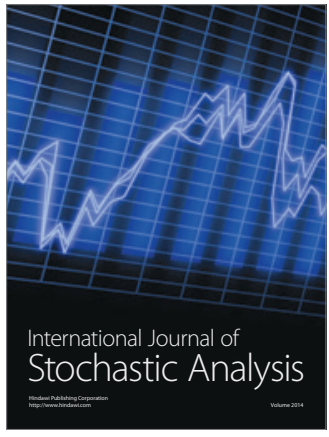

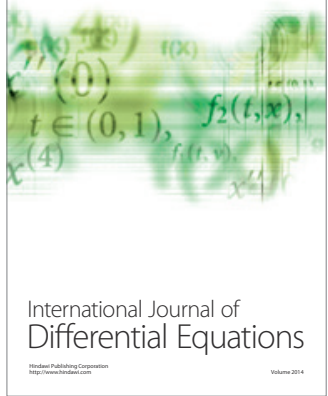
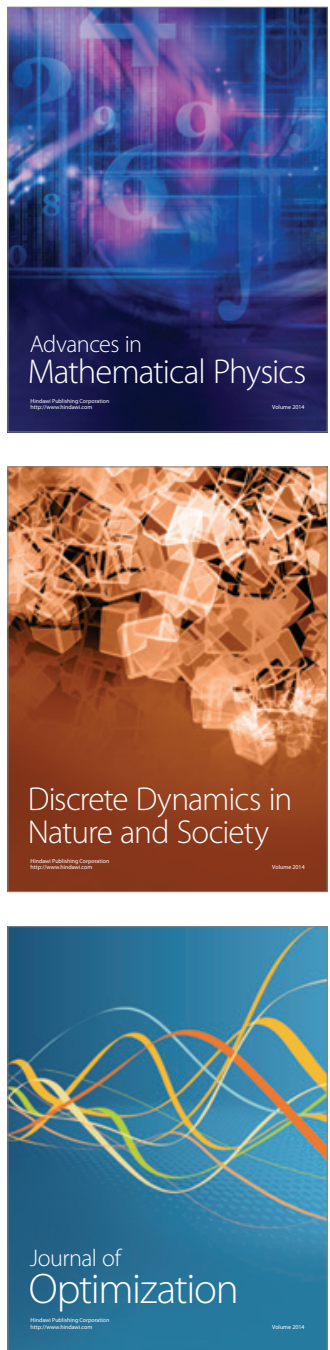